\section{Concentrated surfactant solutions}

The interactions between aggregates can play an important role in more concentrated solutions. Interactions can affect both size and polydispersity of the micelles. They can also promote transitions from spherical to cylindrical micelles in ionic surfactant solutions, because when $c$ is increased, the ionic concentration is increased and the spontaneous curvature is decreased (see shape of the aggregates). The growth of cylindrical micelles with surfactant concentration can be extremely fast if particular ions are added to CTAB solutions, for instance salicylate ions. When the cylinders are long enough, the micelles can entangle, and the solutions become viscoelastic as semi-dilute polymer solutions.

In still more concentrated solutions, the excluded volume interactions among others, can promote the appearance of liquid crystalline phases. They occur generally in the order hexagonal then lamellar, with sometimes cubic phases before and/or after the hexagonal phase. The cubic phase can be either made of isolated micelles in a cubic lattice, or have a bicontinous structure (such as those described by the Schwartz surface). Nematic and rectangular phases have also been observed. In each phase, the packing conditions are only optimal for a given concentration $c$. In the range of existence of this phase and away from this concentration, there is some degree of geometrical frustation which is released by the production of topological defects. The amount of defects is obviously larger at the phase

Denis Weaire and Stefan Hutzler,

Ireland

There is a long history of interest in soap films, bubbles and foams among leading scientists. A bibliography compiled by Mysels, Shinoda and Frankel in 1959 comprises over three hundred references, spanning many centuries boundaries and could promote the transition between the different phases.

Surfactant aggregates are transient because the surfactant constantly changes between the aggregates and the solvent. The exchange time $\tau$ is related to the solubility of the surfactant in the solvent. When the non-polar part is made of $\mathrm{CH}_{2}$ groups, this solubility is related to the free enthalpy cost $\Delta g$ of transfer of a $\mathrm{CH}_{2}$ group from an alkane environment to an aqueous environment. As the cac, the exchange time $\tau$ decreases by a factor 10 when two $\mathrm{CH}_{2}$ groups are added to the chain. For surfactants with chains of 16 carbons, $\tau$ $\sim 10^{-5}$ seconds. For lipids with two carbon chains, $\tau$ is much larger, and can be up to hours. The rheological behaviour of these systems is strongly affected by the exchanges. An extreme case is the sponge phase for which viscosity is only slightly larger than that of water although the structure is connected over a macroscopic scale. The entangled solutions of giant micelles are also less viscous than expected from their equilibrium length.

\section{Polymerization in surfactant systems}

Many attempts to use surfactant structures as templates for the synthesis of small colloidal particles and mesoporous materials have been made in the past. The main problem encountered is that first, the monomers introduced in the systems might adsorb at the surface of the aggregates and change therefore their curvature. The change of spontaneous curvature can also be produced by the polymer, once formed. The polymer also strongly affects the elastic bending moduli of the surfactant layers. Changes in spontaneous curvature and in bending moduli produced by polymers are dependent on the way in which the polymer is connected to the layer: anchored, adsorbed, etc. Electrostatic interactions also play an important role in the complexation of surfactant aggregates and polyelectrolytes with charges of opposite signs.

The study of aqueous mixed solutions of polymers and surfactants is itself presently a very active field of research.

In conclusion, we have briefly described several key ideas in the understanding of structure and dynamic properties of surfactant solutions. The structure properties are now relatively well understood, but more work is needed for a better understanding of the dynamic properties, the rheology in particular. The field of mixed polymer-surfactant systems is expanding, with promising developments both for practical applications and biology.

The author is a research director of CNRS based at the University of Paris South

\section{Further reading}

Micelles, Membranes, Microemulsions and Monolayers edited by W.M. Gelbart, A. Ben-Shaul, D. Roux (Springer, New York, 1994) • D. Langevin Current Opinion in Colloid \& Interface Science 3600 (1998) • Mesoporous Molecular Sieves 1998 edited by L. Bonneviot, F. Beland, C. Danumah, S. Giasson, S. Kaliaguine (Elsevier, Amsterdam, 1998) • Interaction of Surfactants with Polymers and Proteins E.D. Goddard, K.P. Ananthapadmanabhan (CRC Press, Boca Raton, 1993)

\title{
Making, Modelling and Measuring Foams
}

$T_{\text {commended them to poets and }}^{\text {he ephemeral nature of bubbles has }}$ prophets in every age. The ambitious student of the physics of foams should recall Shakespeare's characterisation of the young man seeking "the bubble reputation, even in the cannon's mouth".

Connotations of mortality are compounded with the attractive visual aspect of bubbles in many famous pictures, of which a selection is presented by Michele Emmer in his delightful book Bolle di Sapone: un viaggio tra arte, scienza a fantasia (which awaits, and deserves, a republication in English.)

In this century, scientific interest in bubbles at first receded-Sir James Dewar was one of the last of the old genera- tion to study them. Industrial companies recognized the practical importance of foams and applied research, in the spirit of engineering, developed on a wide front. The books and papers from that mid-century period offer a plethora of empirical data but only minimal insight at a basic level.

Recently, we have begun to rectify this imbalance. Specialized foam conferences have appeared. The proceedings of the most recent meeting in Cargese have just been published by Kluwer. At least two physics networks under the TMR programme and its predecessors have brought together small groups from throughout the European Union, and there have been contributions from East- 

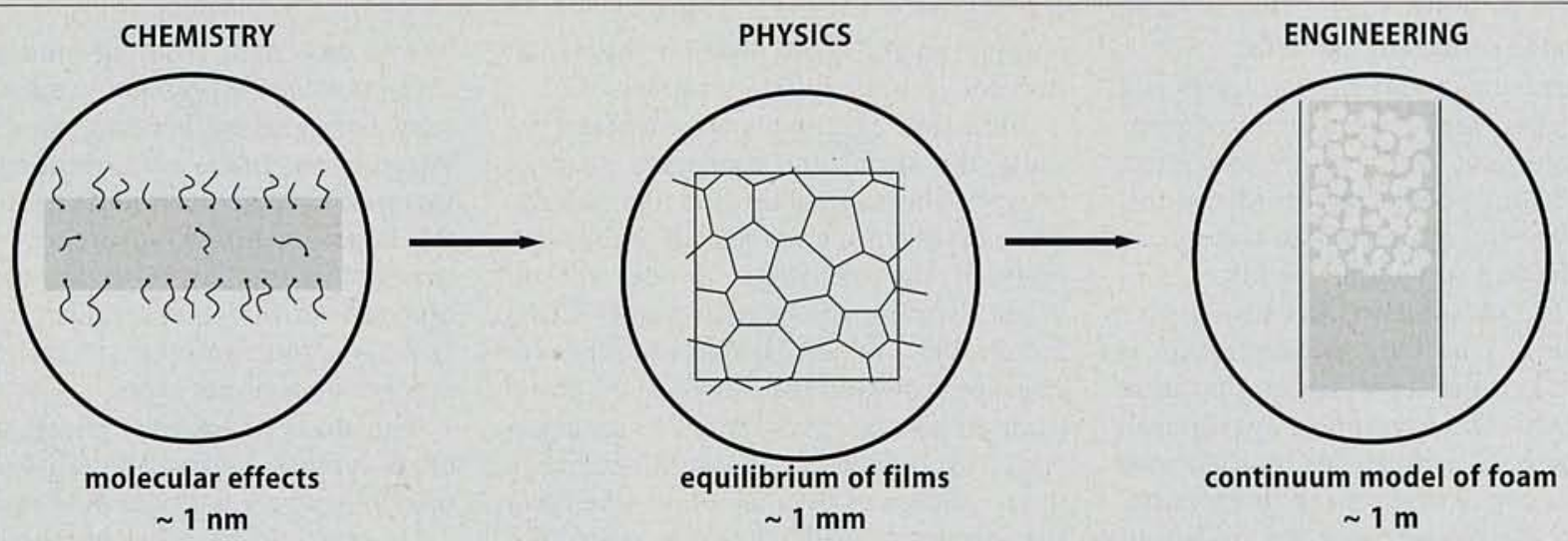

Fig 1 The study of foams is a highly interdisciplinary field of research practised on several different length scales

ern Europe as well. Recently the European Space Agency (ESA) set up a Topical Team to address this area of research and catalyse the development of proposals for foam experiments in the microgravity environment of space.

Most of this new research is devoted to liquid foams and related problems of thin film physics, but it has begun to intersect with the technology of solid foam formation, of equal commercial importance.

\section{Making foams}

A foam may be made by shaking or beating a liquid, by injecting gas in a variety of ways, or by the generation of bubbles from dissolved gas. Immediate solidification, in suitable cases, yields a solid foam. Making films and foams is a game that anyone can play (and most of us do, in the privacy of the bathroom). At an elementary level it is ideal for school projects and impoverished undergraduate teaching laboratories (see A Child's View of Foam by Marc In het Panhuis Europhysics News 29121 1998). It requires little in resources, beyond some simple glassware and a bottle of dishwashing detergent, to make a start. For this reason, bubbles and soap films have always been popular ingredients of the public lecture demonstration, in a tradition dating back to C.V. Boys at the turn of the century. It continues today with Cyril Isenberg, and Gøran Rømme who has just published an account of his own very original and whimsical demonstrations.

More solemn investigations of foam physics do not necessarily require much greater resources. For many of the Physical Review Letters published on foam experiments, the highest cost encountered must have been the publisher's page charges.

Many of the most illuminating experiments such as those of James Glazier and Joel Stavans, as graduate students at the University of Chicago in the 1980s, were carried out on two-dimensional foams between two glass plates. (In keeping with the frugality of this subject, the two young men collected their data on a photocopier.)

Of course, physicists have an inexhaustible appetite for advanced instrumentation, and the experimentation is not all of the kitchen-sink variety. The corresponding theory ranges from applications of simple ideas of classical physics to quite demanding simulations on supercomputers.

A freshly formed liquid foam is subject

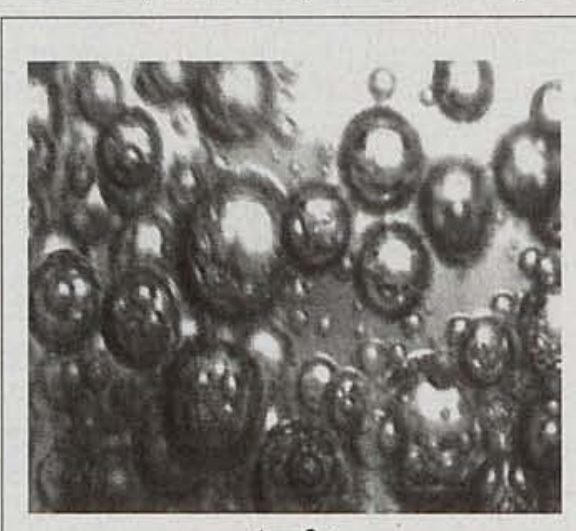

$t=0 s$

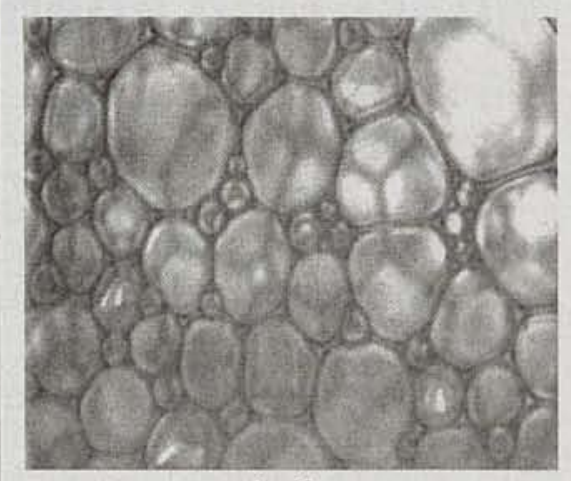

$$
t=2 s
$$

to three processes of development and decay to establish equilibrium under gravity: drainage of the excess liquid, coarsening, and eventual collapse due to rupture of the thin films.

The coarsening process, due to diffusion of gas, attracted the attention of the metallurgist Cyril Stanley Smith to the study of foam around 1950, and his work was the substrate upon which much modern research was eventually based. Drainage is a more recent preoccupation, and attention is now turning to foam collapse.

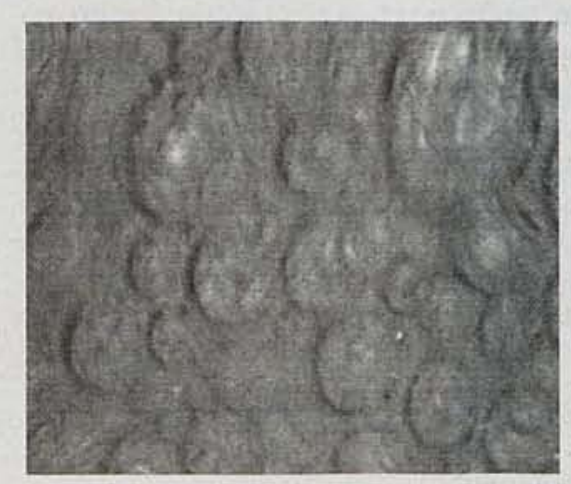
$t=1 s$

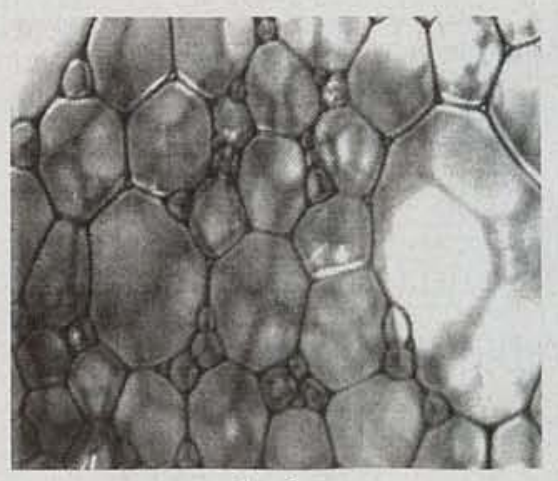

$\mathrm{t}=3 \mathrm{~s}$

Fig 2 Switching on gravity to make a dry foam, in the experiment of C. Monnereau and M. Vignes-Adler, Laboratoires des Phenomènes de Transport dans les Melages du CNRS, Meudon

Source: Influence of Gravity on Foams by C. Monnereau, M. Vignes-Adler and K. Krongerg Journalde Chimie Physique (to appear IN JUNE 1999) 


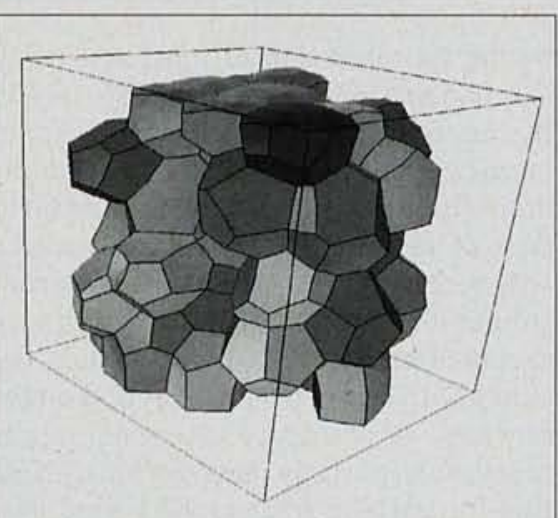

Fig 3 Reconstruction of a bulk foam structure, by C. Monnereau, M. VignesAdler

SOURCE: Optical Tomography Of REAL ThreE-Dimensional

FOAMS JOURNAL OF COLLPID INTERFACE SCIENCE 202 45-53 1998

Research is also moving in the direction of wet foams, those of high liquid fraction, as established by the continuous input of liquid or the use of microgravity.

\section{Modelling foams}

The physicist's approach is to frame an idealized model, in terms of surface tension, and ignore much subtle chemistry at the microscopic level, which may yet come back to haunt us. So far, the bold assumptions of such a model seem to be fully vindicated by the results.

The rules that define such a model for static equilibrium date back to the work of the blind Belgian scientist Joseph Plateau, whose book is one of our main historical landmarks. Both two-dimensional and three-dimensional dry and wet foams can now be simulated in such a model, but dynamic effects cannot yet be reliably included.

\section{Wet and dry foams}

The difference between wet and dry foams is neatly illustrated by the experiments of Claire Monnereau and Michele Adler in airplane flights (in which the effective gravitational field is varied). In sequences of photographs we see bubbles coming together, as gravity is increased from zero (see figure 2 ).

\section{Looking into foams}

It is possible to peer into a very dry foam and admire the curved polyhedral thin films within it. Straightforward video or photography, capturing images from various angles, has been used to generate three-dimensional reconstructions of the foam structure. The reconstruction (figure 3) is accomplished by Monnereau and Adler using the Surface Evolver. This suite of simulation software has been developed by Ken Brakke and collaborators over recent years and is fast becoming a standard tool of foam research. It can generate foam structures, including wet ones, under various constraints and boundary conditions.

This kind of optical imaging fails for wet foams and most solid foams, because of light scattering or opaqueness. If light scattering is dominant all is not lost, in terms of information. As the Exxon group has shown, much can be extracted from measurements of the scattered light, regarding the scale of the structure and the changes taking place within it.

Alternatively, and necessarily in opaque foams, there are other forms of tomography that may be applied. Magnetic Resonance Imaging (MRI), now familiar in the medical context, is increasingly available for condensed matter research. X-ray tomography is another practical alternative and has been applied for example to image metallic foam sandwiches (see John Banhart, Europhysics News 3017 (1999).

If all that is required is the relative density of the foam, electrical resistance provides a convenient probe. Developed for liquid foams at the Shell Laboratories, the resistance profile monitor can record the evolution of the density profile as a foam is wetted or drained.

Such techniques can offer much more than the relatively crude "foamability tests" and other evaluations presently used by industry. In common with colloidal and granular physics, the science of foams, once perplexing in its complexity, is finally becoming more transparent.

The authors are working at Trinity College Dublin

\section{Further reading}

Soap Films by K.J. Mysels, K. Shinoda and S.

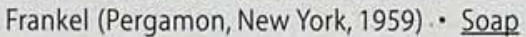
Bubbles in Art and Education by G. Römme (Science Culture Technology Publishing, Singapore, 1998) - Foams and Emulsions edited by S.F.

Sadoc, N. Rivier (Kluwer, 1999) - The Physics of Foams D. Weaire, S. Hutzler (Oxford University

Press) - International Workshop on Foams and Films March 1999 edited by Denis Weaire and John Banhart (Bremen, MIT, 1999)

Research support of Enterprise Ireland and Shell (Amsterdam) is acknowledged

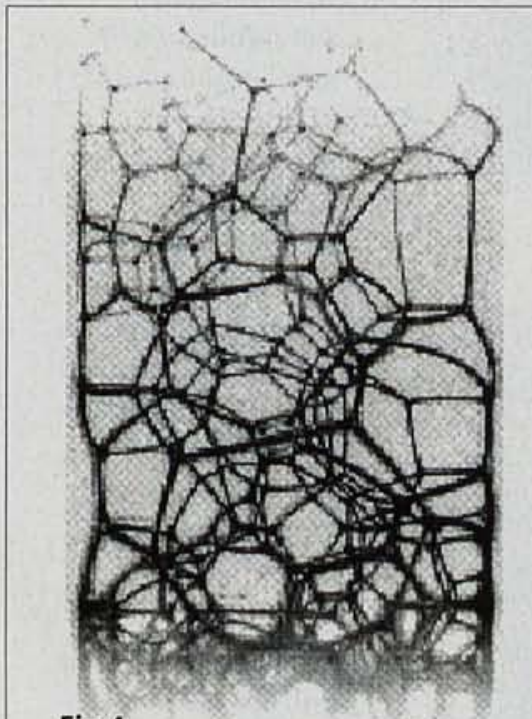

Fig 4

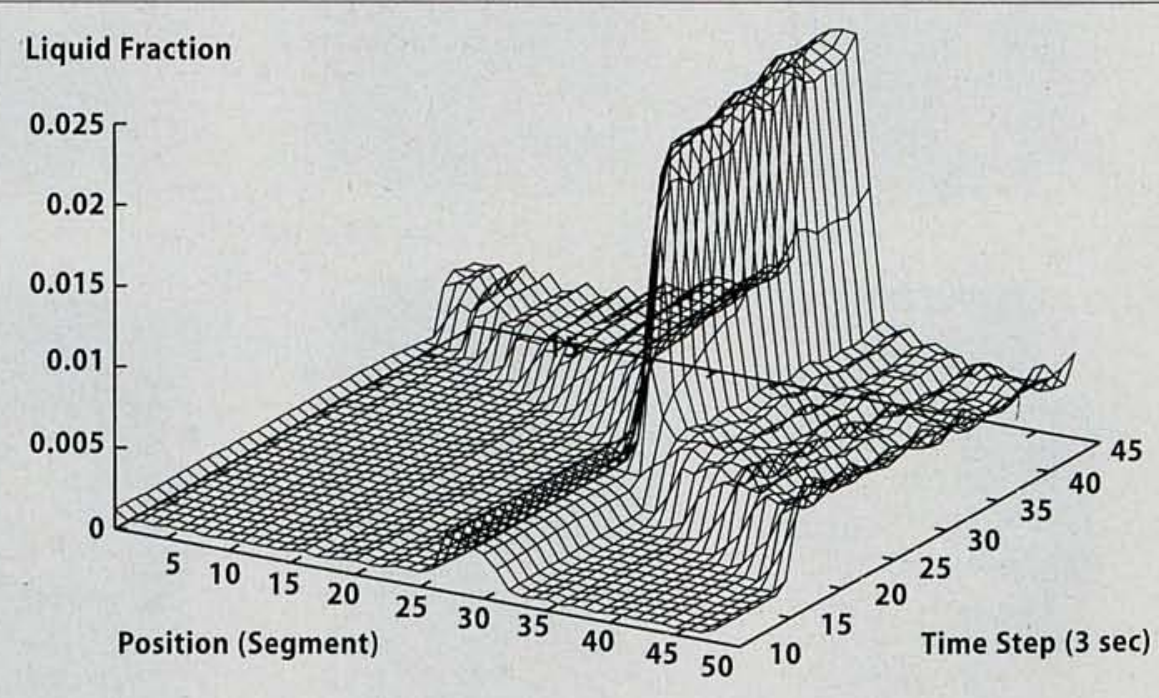

Fig 5

Fig 4 MRI image of a liquid foam - Fig 5 An example of recent data on forced drainage of a non-uniform foam 